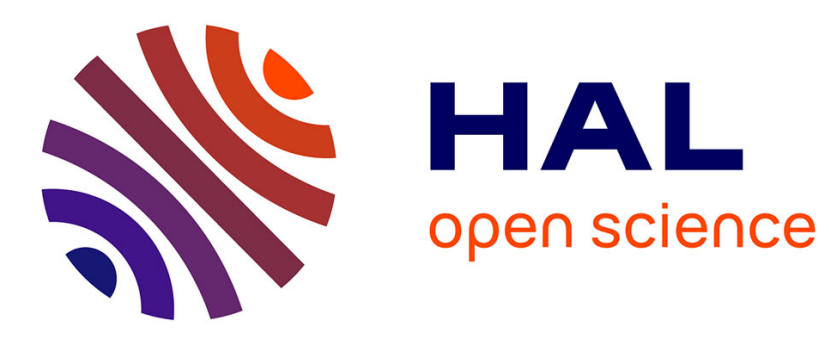

\title{
Reactive Collision Avoidance for Unmanned Aerial Vehicles using Doppler Radar
}

Andrew Viquerat, Lachlan Blackhall, Alistair Reid, Salah Sukkarieh, Graham Brooker

\section{- To cite this version:}

Andrew Viquerat, Lachlan Blackhall, Alistair Reid, Salah Sukkarieh, Graham Brooker. Reactive Collision Avoidance for Unmanned Aerial Vehicles using Doppler Radar. 6th International Conference on Field and Service Robotics - FSR 2007, Jul 2007, Chamonix, France. inria-00195933

\section{HAL Id: inria-00195933 https://hal.inria.fr/inria-00195933}

Submitted on 11 Dec 2007

HAL is a multi-disciplinary open access archive for the deposit and dissemination of scientific research documents, whether they are published or not. The documents may come from teaching and research institutions in France or abroad, or from public or private research centers.
L'archive ouverte pluridisciplinaire HAL, est destinée au dépôt et à la diffusion de documents scientifiques de niveau recherche, publiés ou non, émanant des établissements d'enseignement et de recherche français ou étrangers, des laboratoires publics ou privés. 


\title{
Reactive Collision Avoidance for Unmanned Aerial Vehicles using Doppler Radar
}

\author{
Andrew Viquerat ${ }^{1}$, Lachlan Blackhall ${ }^{1}$, Alistair Reid ${ }^{1}$, Salah Sukkarieh ${ }^{1}$, \\ and Graham Brooker ${ }^{1}$ \\ ARC Centre of Excellence for Autonomous Systems, \\ School of Aerospace, Mechanical and Mechatronic Engineering, \\ The University of Sydney, NSW, 2006, Australia. \\ viquerat@gmail.com, lachlanblackhall@gmail.com, alistaireid@hotmail.com, \\ s.sukkarieh@cas.edu.au, g.brooker@cas.edu.au
}

Summary. Research into reactive collision avoidance for unmanned aerial vehicles has been conducted on unmanned terrestrial and mini aerial vehicles utilising active Doppler radar obstacle detection sensors. Flight tests conducted by flying a mini UAV at an obstacle have confirmed that a simple reactive collision avoidance algorithm enables aerial vehicles to autonomously avoid obstacles. This builds upon simulation work and results obtained using a terrestrial vehicle that had already confirmed that active sensors and a reactive collision avoidance algorithm are able to successfully find a collision free path through an obstacle field.

\section{Introduction}

The ability to deploy UAVs across a multitude of operating environments and mission scenarios has rendered them exceedingly useful for both defence and commercial applications and this has fuelled their rapid development in the last decade. Collision avoidance is a critical autonomous behaviour that is required to achieve a suitably robust platform for mission deployment.

Collision avoidance systems require both obstacle detection sensors and a collision avoidance algorithm that utilises the information obtained from the sensor(s) to determine a path through the obstacle field. Sensors used in existing collision avoidance systems can be broken into two types; active and passive. Examples of passive sensors include optical flow sensors or CCD devices with embedded optical flow algorithms [1] [2], monocular or stereo vision systems using object detection and/or extraction [3] [4] and infrared cameras, which can be used to identify obstacles by the thermal wavelengths they emit. Active sensors include ultrasonic devices, SOund Navigation And Ranging (SONAR), active infrared devices, Laser [5], Doppler measurement radar, conventional radar and Ultra Wideband (UWB) radar [6] [7]. With the 
exception of IR sensors which rely on radiation emitted by the object due to its temperature, any form of passive system requires an external lighting source, which may limit operation in low lighting or low contrast environments. Both Optical flow and Doppler radar sensors require relative motion between the vehicle and target to detect obstacles. As the UAV is continually moving this is not considered a limitation. The angular resolution of the sensors mentioned above varies and is a key consideration in the design process. In selecting a suitable sensor for obstacle detection it is imperative that the size, speed, onboard power resources and payload capabilities of the vehicle are considered. Environmental factors such as the available light and obstacle density also impact upon the choice of a suitable sensor system.

Current collision avoidance methods have utilised a variety of algorithms. Sampling based path planners, which include probabilistic roadmap algorithms and rapidly random trees [8] [9], reactive collision avoidance based on optical flow [1] [2] [5], occupancy grids [10] and receding horizon [11] collision avoidance algorithms have all been used with some success.

\section{Test Vehicles for Collision Avoidance}

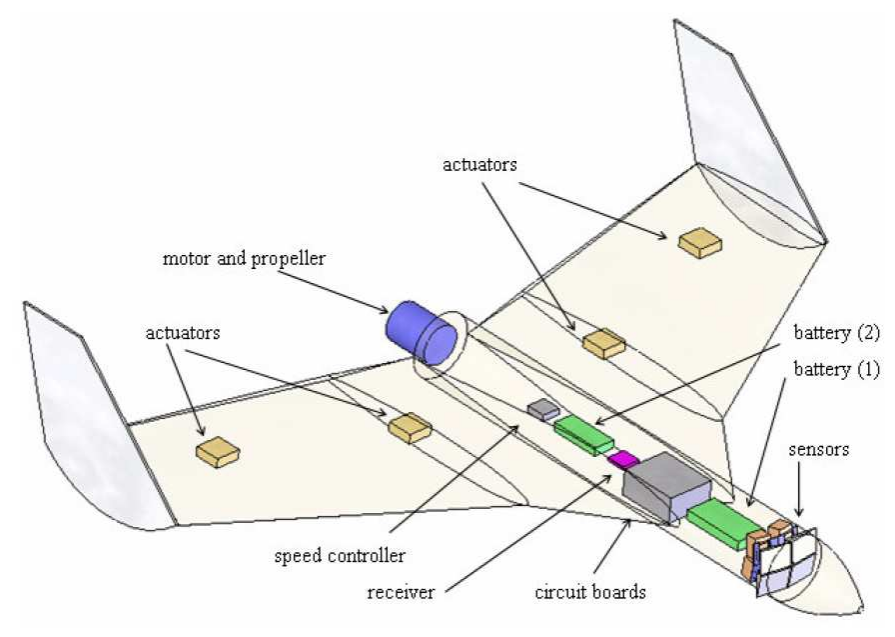

Fig. 1. The UAV designed and built for this project has a mass of $1.503 \mathrm{~kg}$ (Airframe, wingtips and control surfaces 566.3g, Actuators \& actuator arms 4 x 23g, Motor and propeller 213.7g, Speed controller 42g, Receiver 20g, Sensors 4 x $35.8 \mathrm{~g}$, Circuit Boards 161g, Battery (1) 180g, Battery (2) 85g). (Image courtesy of Alex Davidson)

A terrestrial test vehicle allowed preliminary testing and validation of the obstacle detection sensors and the collision avoidance algorithm before im- 
plementation on a UAV. A remote control car was augmented to allow the collision avoidance algorithm to control the steering mechanism while the throttle was controlled via the remote. The collision avoidance algorithm did not have control of the throttle because on the equivalent fixed wing aerial vehicle it is not possible to slow down in the presence of obstacles without changing the flight conditions and risking the initiation of a stall.

The second vehicle to be tested was a mini UAV. This UAV has large control surfaces to facilitate rapid collision avoidance and is shown in Fig. 1.

\section{Obstacle Detection Sensors}

Continuous wave microwave Doppler radars utilising the synthetic aperture technique of Doppler beam-sharpening are ideal for this application. For the aircraft above, the radar unit was developed to have a mass of $304 \mathrm{~g}$, use less than $5 \mathrm{~W}$ and be able to detect the presence of an obstacle at $10-15 \mathrm{~m}(1-2 \mathrm{~s}$ of flight time) with an operation rate of $10 \mathrm{~Hz}$. It was also desired that the angular resolution be less than 20 degrees.

Pulsed Doppler radar can be used to give target velocity, range and in some cases angular location with respect to the observer. Existing work has focussed on the use of this technology for improved automobile safety. Arguably the most successful vehicular collision warning system to date is VORAD (Vehicle On-board RADar) based on the work of Woll [12]. The VORAD system operates at $24.125 \mathrm{GHz}$ to overcome atmospheric attenuation and performs a 4096 point Fast Fourier Transform (FFT), giving an obstacle detection update rate of between 15 and $20 \mathrm{~Hz}$. A large horn antenna (approx 20x14cm) with a gain of $30 \mathrm{~dB}$ and a $-3 \mathrm{~dB}$ beamwidth of $4^{\circ}$ allows a highly directional radar beam that consumes only $0.5 \mathrm{~mW}$ as compared to the $5 \mathrm{~mW}$ transmitters used in this project. To the authors knowledge, no UAV collision avoidance system to date has made use of a Doppler radar obstacle detection sensor system.

\section{Operation of the Doppler Radar Units}

The approximate formula for the Doppler shift which occurs when a target moves much slower than the speed of the reflected wave is given by:

$$
f_{d}=\frac{2 v_{t}}{\lambda} \cos \theta
$$

This can be rearranged to find the angle which the target forms to the radar's velocity vector:

$$
\theta=\cos ^{-1}\left(\frac{f_{d} \lambda}{2 v_{t}}\right)
$$


Note that this equation requires the difference on speed between a target and a vehicle to be known. Here, we assume that all targets are stationary, with a moving vehicle-mounted radar. By measuring the airspeed of the UAV it is possible to ascertain the relative speed between the UAV and a stationary target.

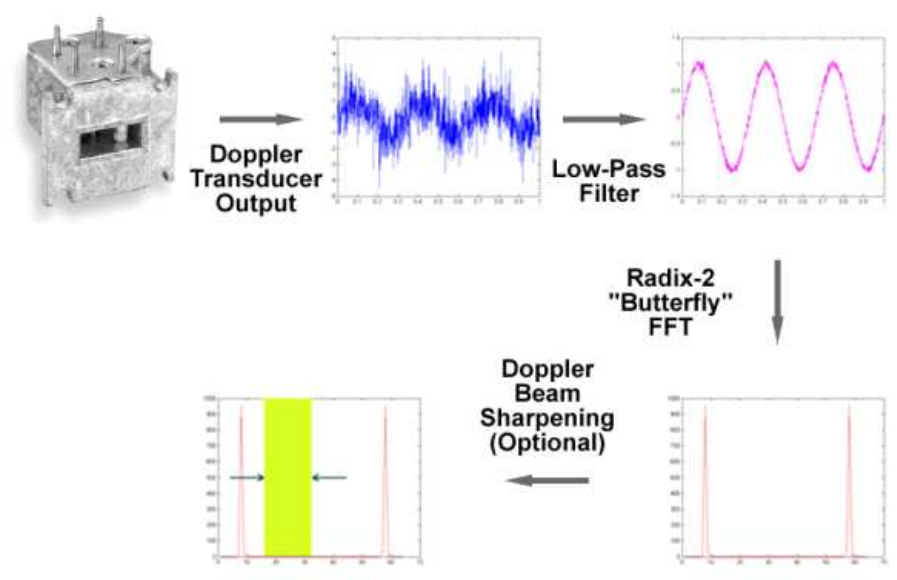

Fig. 2. Operation of the Doppler radar obstacle detection sensors.

The system implemented for this project consists of four microwave Doppler transducers mounted to illuminate the four quadrants of the forward field of view of the UAV. The reason for this will become apparent following the discussion of the reactive collision avoidance algorithm in Section 6 . The output from these sensors is digitally sampled and a 64 point FFT with fixed sampling rate is run over each waveform allowing the peak frequency in each of the radar outputs to be determined [13]. A Pitot-static tube linked to a Micro-Electro-Mechanical Systems (MEMS) pressure sensor allowed the airspeed to be determined, providing the relative speed of the UAV to a stationary obstacle to be determined. Knowledge of the radar parameters and the vehicle speed allow the presence of obstacles in each of the four quadrants to be determined. The use of Doppler beam sharpening allows readings that correspond to obstacles which do not pose a threat to the vehicle's current trajectory to be discarded.

The system can be dynamically configured to operate with or without Doppler beam sharpening allowing the benefits of the synthetic aperture technique to be evaluated. 


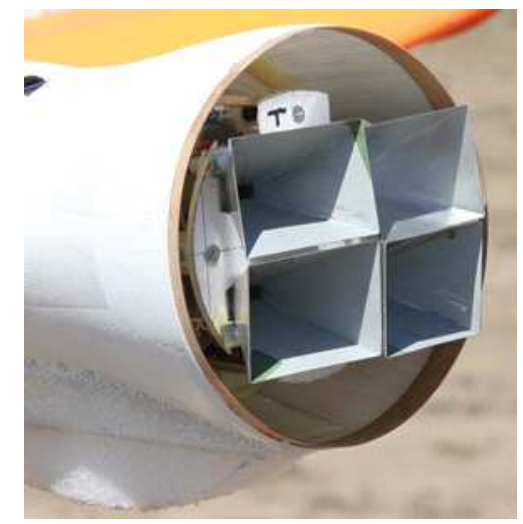

Fig. 3. The antenna configuration used within the UAV allows four individual radar units to monitor the flight path of the UAV for obstacles.

\section{Results and Discussion of the Doppler Radar Units}

Initial tests on the Doppler transducers have demonstrated that the digital sampling and FFT were able to detect the presence of obstacles in the path of the radar units. The features of these units are summarised in Table 1. The update rate and accuracy of the Doppler radar are competing requirements for a given processing power and thus $10 \mathrm{~Hz}$ and a $64 \mathrm{bit} \mathrm{FFT}$ provide a suitable trade off between the demands.

Table 1. Final Doppler radar sensor suite specifications.

\begin{tabular}{ll}
\hline Parameter & Specification \\
\hline Power & $3.7 \mathrm{~W}$ \\
Weight & $304 \mathrm{~g}(382 \mathrm{~g}$ with sensor mount for UAV nose) \\
Radar Dome Diameter & $13 \mathrm{~cm}$ \\
Range & $10-15 \mathrm{~m}$ for a large tree trunk or wall \\
Target Angular Resolution & $15^{\circ}$ \\
Update Rate & $10 \mathrm{~Hz}(7 \mathrm{~Hz}$ in beam-sharpening mode) \\
Cost (No Labour) & $\$ 500(\mathrm{AUD})$ \\
\hline
\end{tabular}

Testing of the radar units progressed in two phases. The radar units were first tested in isolation with relative motion between the unit and a reflecting target. These tests ascertained that the size of the FFT was adequate for this purpose. In addition, airflow of known velocity was used to verify the operation of the Doppler beam-sharpening.

The second phase involved the use of the system (with only two radars operating) in an obstacle detection application mounted on a small remote 
controlled terrestrial vehicle. The tests were performed without Doppler beamsharpening and full discussion and analysis can be found in Section 7 .

An obvious assertion that must be made about Doppler radar is that the magnitude of the return signal is dependant on both the size and material type of the obstacle[14] [15]. For this reason the range will be dependant on the environment in which the radar system is used, and the type of obstacles the UAV will encounter. However, it must be remembered that the obstacles we expect to encounter (trees, buildings \& the ground) will all provide a significant magnitude of radar return and thus the use of Doppler radar for obstacle detection is appropriate.

\section{A Reactive Collision Avoidance Algorithm}

The purpose of the collision avoidance system is to find a continuous, unimpeded trajectory autonomously. At each point in time, the attainable flight trajectories of the UAV are determined by manoeuvrability and flight speed constraints. The region of space monitored by the radar units is divided into a series of sub-regions (Fig. 4), each corresponding to a different trajectory direction. The authors propose the use of the term activation to describe the danger of flying into a given sub-region, where a higher activation corresponds to more or larger obstacles. For this purpose, the obstacle detection sensors must return higher magnitude values for closer and denser obstacles.

The measured radar returns are attributed to the sub-region from which they are taken using statistical estimation theory and the trajectory for the next time step is determined by choosing the sub-region with the lowest activation. In this application, the algorithm is used every $0.1 \mathrm{~s}$, which corresponds to the update rate of the Doppler radar units.

In this application, four dedicated Doppler radar units monitor the region in front of the UAV. The reactive algorithm will guide the UAV into the region monitored by the Doppler radar unit with the lowest return radar signal. By choosing the field of view of the sensors sufficiently wide it is considered unlikely that the collision avoidance algorithm will choose a trajectory towards a previously avoided obstacle. As the algorithm is calculating the trajectory at each point in time based only on the current sensor readings, it is considered to be making stateless decisions.

Existing collision avoidance systems have typically focused on stateful collision avoidance. The authors propose a new approach where the collision avoidance system is stateless, because stateless machines are capable of rapid analysis of time variant data that does not exhibit strong correlation.

For a UAV in a dense obstacle field, it is probable that the ability of the collision avoidance system to predict the obstacle field of a given trajectory

will be poor, due to the limited computing power available coupled with the highly dynamic motion of the platform. This dynamic motion is likely to be 


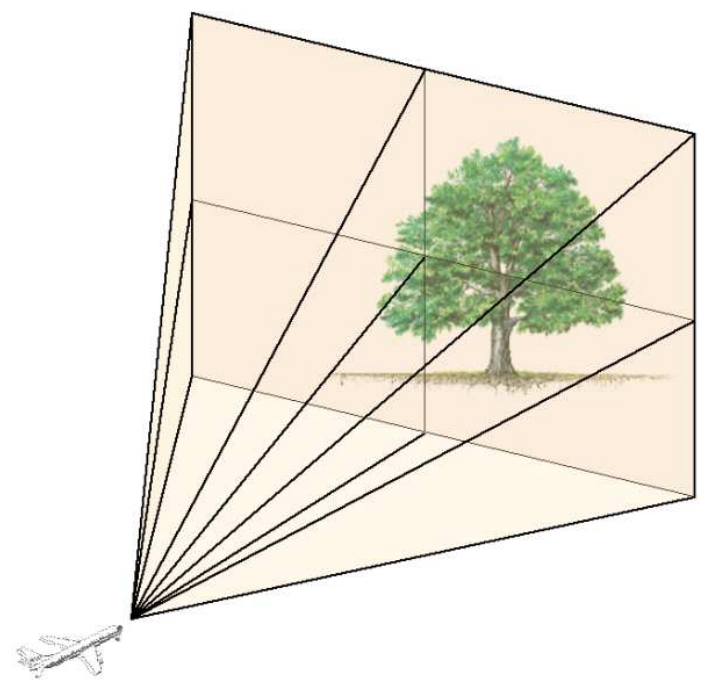

Fig. 4. The forward image plane decomposed into quadrants. Each quadrant is monitored for the presence of obstacles by a dedicated Doppler radar unit.

even more apparent on mini and micro air vehicles, because aerodynamic effects and manoeuvre behaviours are amplified by the low wing loading and the small inertial resistance provided by the airframe to motion. This would suggest that a purely reactive collision avoidance system could provide computational benefits over existing collision avoidance algorithms and implementations, without significant performance compromises.

It must be noted that the reactive nature of the collision avoidance system means that if a continuous unimpeded path does not exist then the algorithm will result in the loss of the UAV as the algorithm uses only the sensor readings at each time step in choosing the best trajectory for the next period of flight time.

\section{Experimental Results and Discussion}

Preliminary results were obtained using a remote control car fitted with the collision avoidance system (utilising only two obstacle detection radar units). The vehicle was driven directly towards an obstacle approximately $60 \mathrm{~cm}$ in diameter. In these tests, the vehicle was traveling about $1 \mathrm{~m} / \mathrm{s}$ and was successfully able to avoid the obstacle. Figure 5 shows the vehicle steering right to avoid the obstacle, as the right radar unit registered a lower activation than the left, and hence a reduced danger of collision.

Further tests on the ground vehicle have confirmed that this algorithm is able to navigate through a cluttered obstacle field in real-time despite its 


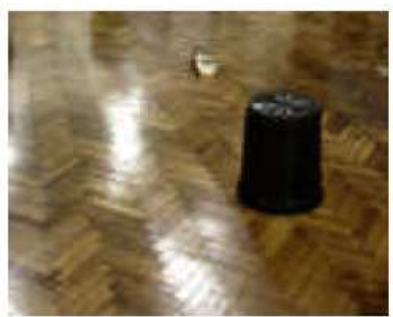

(a)

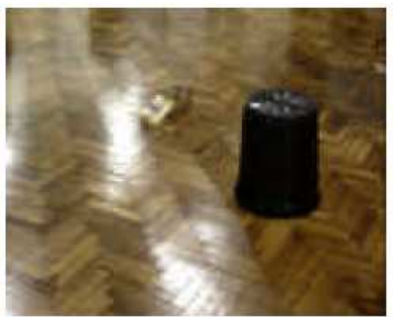

(b)

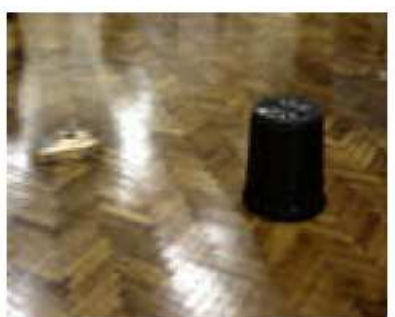

(c)

Fig. 5. The terrestrial test vehicle avoiding an obstacle in its path using Doppler radar obstacle detection sensors and a reactive collision avoidance algorithm.

limited computational resources. Similar trials are yet to be shown on the air vehicle.

To validate the functionality of the collision avoidance algorithm and sensor suite, the UAV discussed previously was flown towards an obstacle and the resulting trajectory observed. The obstacle utilised was a tethered radar reflector. The reflector was designed to minimize risk to the UAV, giving a strong radar reflection and with a low mass in case a collision did occur. In this test the UAV was flown towards the target by a human pilot and then the autonomous mode was activated. Once in autonomous operation the collision avoidance system had control of the ailerons allowing a lateral avoidance manoeuvre to be performed. Photographs of an avoidance manoeuvre are shown in Fig. 6. In this sequence of images the UAV approaches the target, from the left, at a speed of approximately $8 \mathrm{~m} / \mathrm{s}$ and then initiates a collision avoidance manoeuvre to the left to avoid the obstacle. Given the range of the radar units is approximately $10 \mathrm{~m}$, this approach speed gives the UAV a window of approximately one second to complete its avoidance manoeuvre.

The preliminary tests above were designed to validate the methodology of this approach and have demonstrated that reactive collision avoidance has the potential to allow air vehicles, especially those with limited computational resources, to navigate through obstacle fields using Doppler radar units.

While early tests have been successful, additional flight tests need to be conducted to further validate the approach used. A significant limitation observed thus far is the accurate range of the Doppler radar units. An increase in range would increase the manoeuvre horizon (the time allowed for manoeuvres prior to a collision) for a given obstacle allowing the UAV to avoid significantly larger obstacles in the flight path. In the current implementation, noticeable noise is induced in the radar signal by the signal-processing electronics mounted within the UAV. This noise is significantly decreasing the usable range of the units. Increased shielding between the radar units and the other electronics should increase the accurate range of the Dopler radar units allowing this system to be utilised by UAVs with higher operational speeds. 


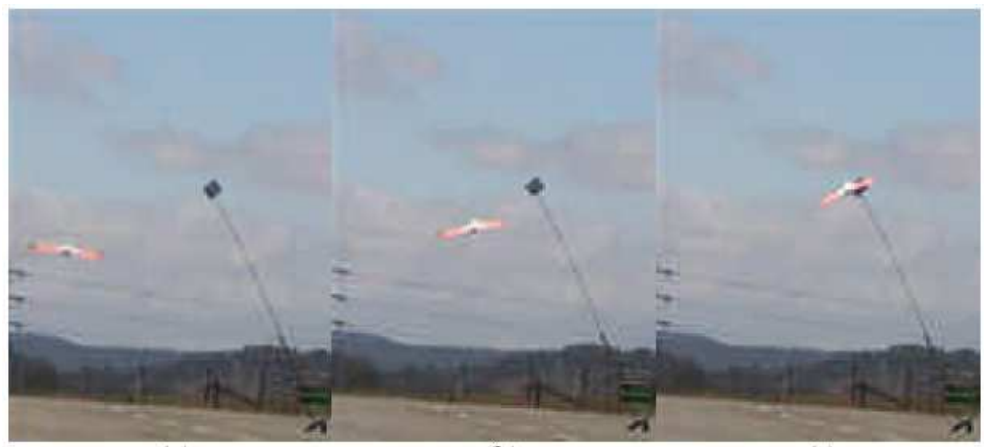

(a)

(b)

(c)

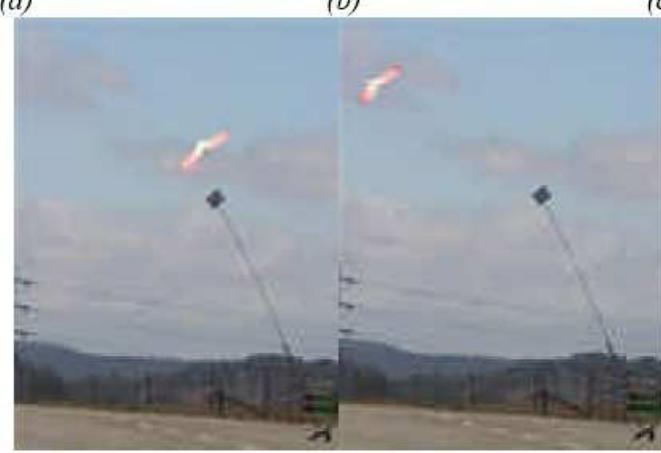

(d)

(e)

Fig. 6. The UAV autonomously avoiding an obstacle by rapidly turning away.

\section{Conclusion}

Existing approaches to collision avoidance have favoured short term dynamic trajectory planning to solve the collision avoidance problem. Unfortunately, such methods become computationally expensive in dense obstacle fields when the collision avoidance system must rapidly process large amounts of data to prevent potential collisions. The fully reactive approach is advantageous because its computational costs are independent of the number of obstacles present in the obstacle field. For this reason, it becomes feasible for aerial platforms of any size. This design shares its philosophy with optical flow sensors, providing effective performance with a low computational complexity.

In addition to improving the radar unit shielding, future work may involve applying the synthetic aperture radar technique of Doppler beam-sharpening. This technique does not rely on any kind of antenna directionality. Rather, it uses velocity components to 'spotlight' obstacles directly in the path of a moving vehicle. A continuous wave microwave Doppler radar system which makes use of Doppler beam-sharpening has potential as the basis for a collision avoidance system in aerial vehicles and further work on the concept is certainly justified. 


\section{Acknowledgements}

This work is supported by the ARC Centres of Excellence programme funded by the Australian Research Council (ARC) and the New South Wales State Government. The authors must thank Mr Jeremy Randle and Mr Alan Trinder who worked tirelessly to ready the UAV for flight trials and also to Dr Hugh Stone and Mr Alex Davidson who undertook the computer modelling and flight simulation of the UAV.

\section{References}

1. Barrows, G. (1999) Mixed-Mode VLSI Optic Flow Sensors For Micro Air Vehicles. PhD thesis, University of Maryland, College Park.

2. Zufferey, J. (2005) Bio-Inspired Vision-Based Flying Robots. PhD thesis, Ecole Polytechnique Federale De Lausanne.

3. McGee, T., Sengupta, R., and Hedrick, K. (2005) Obstacle detection for small autonomous aircraft using sky segmentation. Proceedings of the IEEE International Conference on Robotics and Automation.

4. Lee, D., Beard, R., Merrel, P., and Zhan, P. (2004) See and avoidance behaviors for autonomous navigation. SPIE Opics East, Robotics Technologies and Architectures, Mobile Robot XVI, 5609-05.

5. Griffiths, S., Saunders, J., Curtis, A., McLain, T., and Beard, R. Obstacle and terrain, avoidance for miniature aerial vehicles. IEEE Robotics and Automation Magazine, To Appear.

6. Gresham, I., et al. (2004) Ultra-wideband radar sensors for short-range vehicular applications. Transactions on Microwave Theory and Techniques, 52, 21052122.

7. Fontana, R., Richley, E., Marzullo, A., Beard, L., Mulloy, R., and Knight, E. (2002) An ultra wideband radar for micro air vehicles air vehicle applications. IEEE Conference on Ultra Wideband Systems and Technologies.

8. Lingelbach, F. (2005) Path Planning using Probabilistic Cell Decomposition. $\mathrm{PhD}$ thesis.

9. Pettersson, P. (2006) Sampling-based Path Planning for an Autonomous Helicopter. PhD thesis, Linkoping Institute of Technology at Linkoping University.

10. Sinopoli, B., Micheli, M., Donato, G., and Koo, J. (2001) Vision based navigation for an unmanned air vehicle. Proceedings of the IEEE International Conference on Robotics and Automation, pp. 1757-1765.

11. Frew, E., Langelaan, J., and Joo, S. (2006) Adaptive receding horizon control for vision based navigation of small unmanned aircraft. Proceedings of the 2006 American Control Conference.

12. Woll, J. (1995) Vorad collision warning radar. pp. 369-372.

13. Brigham, E. (1974) The Fast Fourier Transform. Prentice-Hall Inc.

14. Sarabandi, K. and Park, M. (2003) A radar cross-section model for power lines at millimeter-wave frequencies. IEEE Transactions on Antennas and Propagation, 51, 2353-2360.

15. Yonemoto, N., Yamamotoa, K., Yamadaa, K., Yasuib, H., Tanakab, N., Migliaccioc, C., Dauvignacc, J., and Pichotc, C. (2006) Performance of obstacle detection and collision warning system for civil helicopters. Proc. SPIE, 6226. 